\title{
The Management of an Early COVID-19 Case with Severe Acute Respiratory Distress Syndrome in a Community Hospital in On- tario, Canada
}

Maham Khalid, BMSc ${ }^{1}$, Han-Oh Chung, MD, MSc HS Ed ${ }^{1,2,3}$ Dimitra Fleming BSc Phm ${ }^{3}$ George Far jou, $M D^{1,2,3}$ and Jennifer LY Tsang, MD, PhD $D^{1,2,3^{*}}$

${ }^{1}$ Niagara Regional Campus, Michael G. DeGroote School of Medicine, St. Catharines, Ontario, Canada

${ }^{2}$ Department of Medicine, McMaster University, Hamilton, Ontario, Canada

${ }^{3}$ Niagara Health, St. Catharines, Ontario, Canada

*Corresponding author: Jennifer LY Tsang, Niagara Regional Campus, Michael G. DeGroote School of Medicine, St. Catharines, Ontario, Canada; Department of Medicine, McMaster University, Hamilton, Ontario, Canada; Niagara Health, St. Catharines, Ontario, Canada, Tel: 905-378-4647 ext 42919, Fax: 289-398-1059

\begin{abstract}
Background: We reported an early COVID-19 case in a young patient in a Canadian community intensive care unit (ICU) with severe acute respiratory syndrome (ARDS). We discussed the management according to the recently published Surviving Sepsis Guidelines on COVID-19. This will provide critical care physicians important and timely information on the management of severe COVID-19 using a real case when critical care physicians are facing a surge in COVID-19 cases. Many of the recommendations regarding ARDS are extrapolated from previous literature in the critically ill, with new information on the adjunctive use of steroids, antivirals and immunomodulatory therapies now emerging.
\end{abstract}

Case presentation: This is a case of a 39-year-old patient in a community ICU in Southern Ontario, Canada, with severe COVID-19 complicated by circulatory shock requiring vasopressor support; severe ARDS requiring lung protective ventilation, use of a neuromuscular blocking agent, proning, systemic corticosteroids and conservative fluid management; and acute kidney injury requiring intermittent hemodialysis. The management of this patient was guided by the recently published Surviving Sepsis Guidelines on COVID-19. He required vasopressor support for 2 days for circulatory shock. After 12 days of mechanical ventilation, the patient was liberated from the ventilator but remained on intermittent hemodialysis. He was transferred to the medical ward on day 14 of his hospital/ICU admission.

Conclusions: Healthcare professionals in critical care will face a continued surge of critically ill COVID-19 patients with ARDS in the coming weeks to months. Moreover, due to the infectious nature of this disease, inter-hospital transfer of these types of patients for higher levels of care will be limited. As such, both academic and community ICUs must be equipped to manage these critically ill patients with COVID-19.

\section{Keywords}

Novel Coronavirus, SARS CoV-2, COVID-19, ARDS, Critical illness

\section{List of Abbreviations}

ICU: Intensive Care Unit; ARDS: Acute Respiratory Syndrome; WHO: World Health Organization; COVID-19: Novel Corona Virus disease; SSC: Surviving Sepsis Campaign; PEEP: Positive End Expiratory Pressure; $\mathrm{FiO}_{2}$ : Fraction of Inspire Oxygen; NMBA: Neuromuscular Blocking Agent; PCR: Polymerase Chain Reaction; ARB: Angiotensin Receptor Blocker; LTVV: Low Tidal Volume Ventilation; VILI: Ventilator-Induced Lung Injury; ETT: Endotracheal Tube; PPE: Personal Protective Equipment; PROSEVA: Prone Positioning in Severe Acute Respiratory Distress Syndrome; ACE2: Angiotensin Converting Enzyme 2; RAAS: Renin-Angiotensin-Aldosterone System; CATCO: Multi-Center, Adaptive, Randomized, Open-label, Controlled Clinical Trial of the Safety and Efficacy of Investigational Therapeutics for the Treatment of COVID-19 in Hospitalized Patients; ACT: Anti-Coronavirus Therapies to prevent progression of COVID-19; INR: International Normalized Ratio; AST: Aspartate Aminotransferase; ALT: Alanine Aminotransferase; ALP: Alkaline Phosphatase; IFEU: Fibrinogen Equivalent Unit 


\section{Introduction}

In December 2019, there was an emergence of a novel coronavirus that caused a cluster of pneumonia cases in Wuhan, China, now known as the coronavirus disease (COVID-19). The causative virus was identified as SARS-CoV2, a positive single-stranded RNA virus in the same coronavirus family as SARS-CoV and MERSCoV [1]. COVID-19 was declared a pandemic by the World Health Organization (WHO) on March 11, 2020.

As of April 8, 2020, more than 1.4 million cases were confirmed worldwide with over 85,000 deaths [2]. Approximately 5\% of patients with COVID-19 infection progress to critical illness with respiratory failure [3]. Therefore, the number of critically ill patients with COVID-19 who will require intensive care unit (ICU) beds is expected to rise across the globe in the coming weeks. Although novel, there has already been a significant influx of new information and studies from around the world on this new illness. Recently, Canadian authors have made recommendations on the management of critically ill adults with COVID-19 through updates to the Surviving Sepsis Campaign (SSC) [4]. Pertaining specifically to patient care, the guidelines describe several strategies for adult patients with acute respiratory distress syndrome (ARDS) and shock. In the following case study, we discuss the first 12-days of ICU management of a young patient with COVID-19 complicated by ARDS, circulatory shock, and renal failure outlining the practical application of the SSC in a critically ill patient in an urban community hospital in Canada. Informed consent was obtained from the patient and the Hamilton Integrated Research Ethics Board (REB) has waived the requirement of REB approval.

\section{Case Presentation}

In mid-March of 2020, a 39-year-old man was sent home from an inpatient drug rehabilitation center in Southern Ontario after an exposure to a caregiver who was later confirmed to have COVID-19. A day after discharge from the rehabilitation center, the patient experienced fever, chills, headache and shortness of breath. Five days after discharge, he was tested for COVID-19 at an outpatient assessment clinic, then went home to follow self-isolation precautions.

Seven days after discharge from the drug rehabilitation center, the patient was brought to an emergency department of a peripheral hospital in Southern Ontario with a presenting symptom of shortness of breath. On physical examination, he was found to be hypoxemic with an oxygen saturation of $70 \%$ on ambient air. He was placed on supplemental oxygen via Venturi mask at 15 litres/minute with an improvement of oxygen saturation to $90 \%$. His presenting temperature was $37.9^{\circ}$ Celsius. His initial blood pressure was $150 / 75 \mathrm{mmHg}$. He was found to be tachypneic with respiratory rate of 36 breaths per minute and tachycardic with pulse rate of 120 beats per minute. On examination, he was alert with a Glasgow Coma Score of 15, with distant heart sounds and scattered crackles on chest auscultation.

Due to worsening hypoxemia, the patient was intubated in a negative pressure room in the emergency department. A first attempt was made by the respiratory therapist which was unsuccessful. Subsequently, he was intubated by an emergency physician with the use of midazolam (10 mg), propofol (300 mg in divided doses), and succinylcholine (200 $\mathrm{mg}$ in divided doses). Shortly after intubation, he was placed on pressure con-

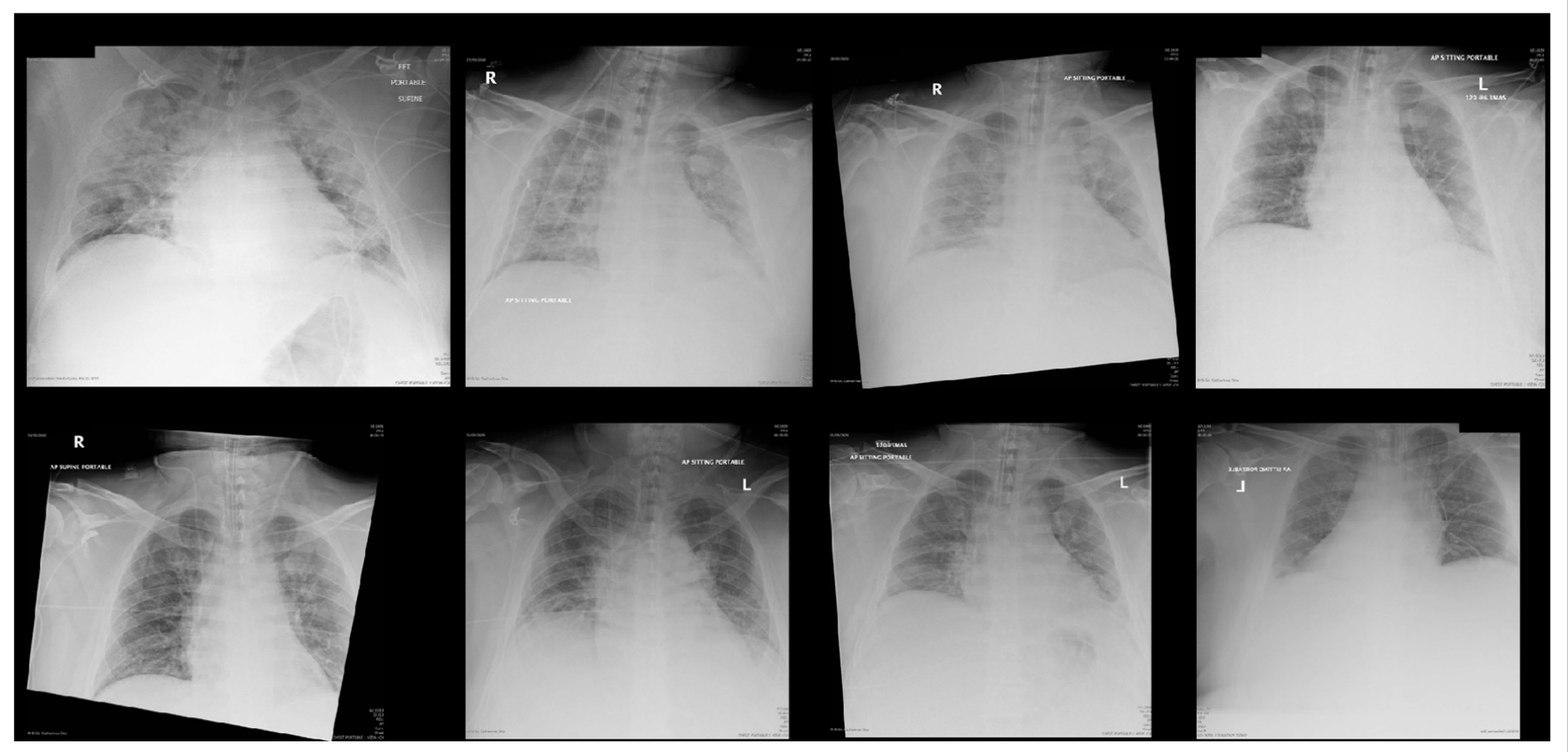

Figure 1: Progression of chest radiographs of 39-year-old patient with SARS-CoV-2. Figures are lettered in consecutive order. A) Illustrates the patient at initial presentation (Day 1); B) taken on Day 3; C) taken on Day 4; D) taken on Day 5; E) taken on Day 6; F) taken on Day 7; G) taken on Day 8; H) taken on Day 9. 
trol ventilation with a driving pressure of $15 \mathrm{cmH}_{2} \mathrm{O}$ and a positive end expiratory pressure (PEEP) of $15 \mathrm{cmH}_{2} \mathrm{O}$. Despite being placed on fraction of inspire oxygen $\left(\mathrm{FiO}_{2}\right)$ of $100 \%$, he remained hypoxemic with oxygen saturation between $70-80 \%$. As such, he was placed on a neuromuscular blocking agent (NMBA) for patient-ventilator asynchrony. He was also started on piperacillin/ tazobactam for community acquired pneumonia.

The patient's medical history included class III obesity, hypertension, depression, anxiety, recreational drug use (cocaine and opioids) and previously undiagnosed diabetes (diagnosed after admission to ICU). His home medications consisted of candesartan, escitalopram, ariprazole and trazodone.

A plain film chest radiograph was performed, which was reported as bilateral alveolar infiltrates with consolidation (Figure 1). His initial leukocyte count was 5,600 millions/L and his lymphocyte count was low at 900 millions/L, with a neutrophil:lymphocyte ratio of 7.54. His initial creatinine was normal at 93 micro$\mathrm{mol} / \mathrm{L}$. His arterial blood gas after intubation showed a pH of 7.19, $\mathrm{pCO}_{2}$ of $84 \mathrm{mmHg}, \mathrm{pO}_{2}$ of $70 \mathrm{mmHg}$. His $\mathrm{PaO}_{2}: \mathrm{FiO}_{2}$ was 70 , meeting criteria for severe disease defined as a $\mathrm{PaO}_{2}: \mathrm{FiO}_{2}$ ratio of $\leq 200$. (see Table 1, for his laboratory values).

A nasopharyngeal swab specimen was obtained in the emergency department and sent for detection of viral respiratory pathogens by real-time polymerase chain reaction (PCR) for SARS-CoV 2 Envelope gene; this was reported back as positive within 24 hours in a laboratory in the local region.

Due to persistent hypoxemia, he was then transferred to the ICU of a dedicated COVID-19 community hospital in the same region on the same day of his emergency room presentation for further care. The patient was placed in a negative pressure room and airborne precautions were undertaken by all healthcare workers.

The patient was hemodynamically unstable initially and required vasopressor support (Norepinephrine 1-8 $\mathrm{mcg} / \mathrm{min}$ ). A conservative fluid strategy was adopted in the management of this patient to maintain the patient near euvolemic status. This patient was weaned off the vasopressor on the second day of ICU admission (See Table 2, for details of his vitals and vasopressor medication).

The patient's course was complicated by evidence of severe ARDS with a $\mathrm{PaO}_{2}: \mathrm{FiO}_{2}$ ratio of 70 . He was deeply sedated with continuous infusion of propofol, midazolam and fentanyl. He was also placed on continuous infusion of a NMBA, cisatracurium, to aid in patient-ventilator synchrony. A recruitment maneuver was employed (PEEP of $40 \mathrm{cmH}_{2} \mathrm{O}$ for 40 seconds). A proning procedure was undertaken daily for 16 hours for four days for severe hypoxemia. It was noteworthy that his
$\mathrm{PaO}_{2}: \mathrm{FiO}_{2}$ ratio improved from 70 to $>200$ when he was placed in prone position and his oxygen requirement decreased from $\mathrm{FiO}_{2}$ of $100 \%$ to $40 \%$. A lung protective ventilatory strategy was employed with high PEEP and low tidal volume (4-8 $\mathrm{mL} / \mathrm{kg}$ predicted body weight). As recommended by the SSC COVID-19 Guidelines, the patient was placed on systemic corticosteroid for ARDS (see Table 3, for details on the ARDS management of this patient).

His course was further complicated by acute kidney injury that necessitated renal replacement therapy in the form of intermittent hemodialysis. From the antimicrobial point of view, he was placed on piperacillin/tazobactam, azithromycin and oseltamivir initially. When his nasopharyngeal test result returned, oseltamivir was discontinued. Subsequently, piperacillin/tazobactam was discontinued, azithromycin was continued for 5 days in total and ceftriaxone was started for 4 days in total. A urinary Legionella antigen test was negative. An endotracheal aspirate was sent for microbiology on his second day of ICU admission and grew Staphylococcus aureus that was methicillin sensitive and cefazolin was started on day 7 of his ICU admission for superimposed bacterial pneumonia (see Table 4, for details of his antimicrobial administration).

Given the uncertainty of the relationship between angiotensin receptor blockers (ARB), in this case, candesartan, and ARDS secondary to COVID-19, the healthcare team did not continue it during his ICU stay. Additionally, the patient was initially hypotensive and suffered from kidney injury which precluded the re-initiation of candesartan.

We followed this patient for the first 12-days of his ICU stay. During this time, his circulatory shock resolved, the severity of his ARDS improved after the use of a NMBA and proning. He was extubated on day 12 successfully and was subsequently transferred out of the ICU. However, he remained on intermittent hemodialysis.

\section{Discussion and Conclusions}

When patients with COVID-19 present to the ICU with respiratory failure, supportive care with mechanical ventilation continues to be the mainstay of treatment. We continued to use low tidal volume ventilation (LTVV) (4-8 mL/kg of predicted body weight) and limiting plateau pressures to $<30 \mathrm{cmH}_{2} \mathrm{O}$ in line with current ARDS management. The SSC COVID-19 Critical Care guidelines continue to endorse this practice due to the large quantity of evidence that points to its benefit in the general ARDS population as well as its low cost and ease of implementation [4]. LTVV [5] along with limiting plateau pressure [6] can reduce ventilator-induced lung injury (VILI) and consequently can reduce associated secondary multiorgan failure of patients with ARDS [7].

Our patient additionally required proning due to se- 


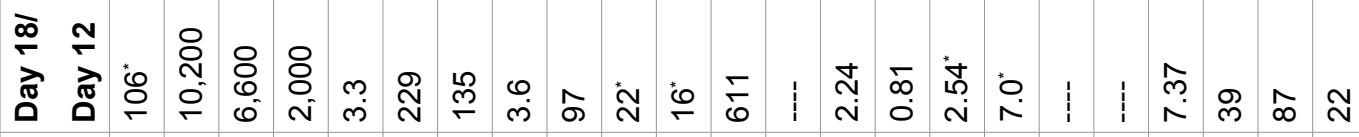

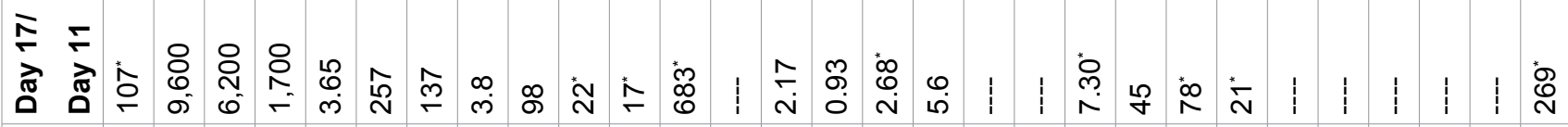

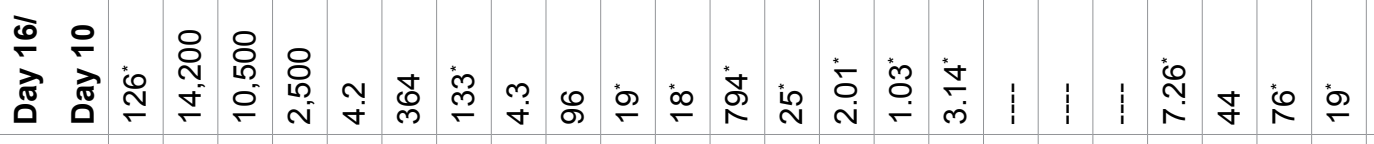

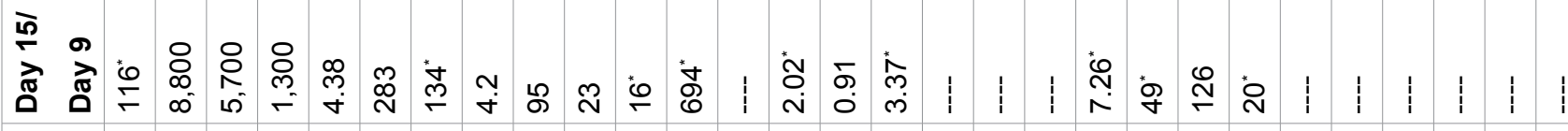

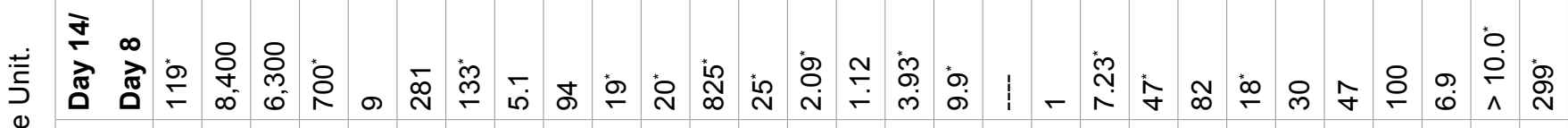

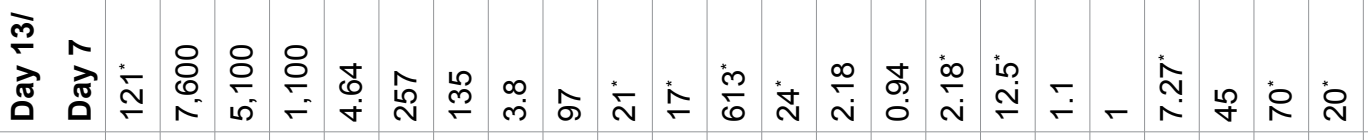
ลิ 产

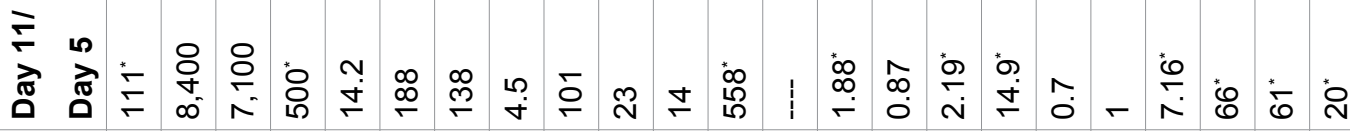
产

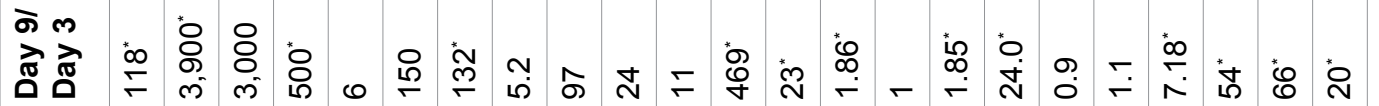

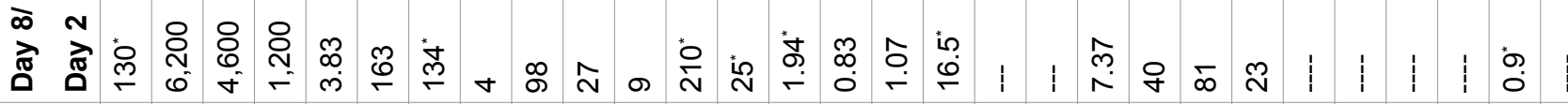

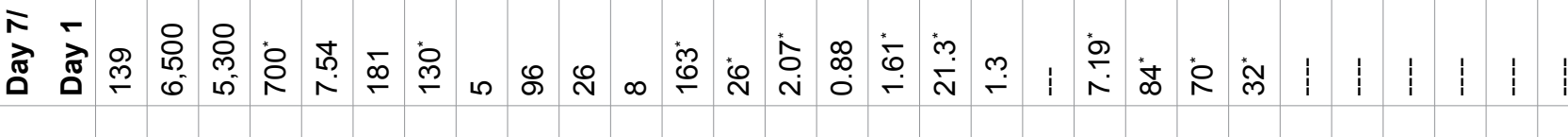

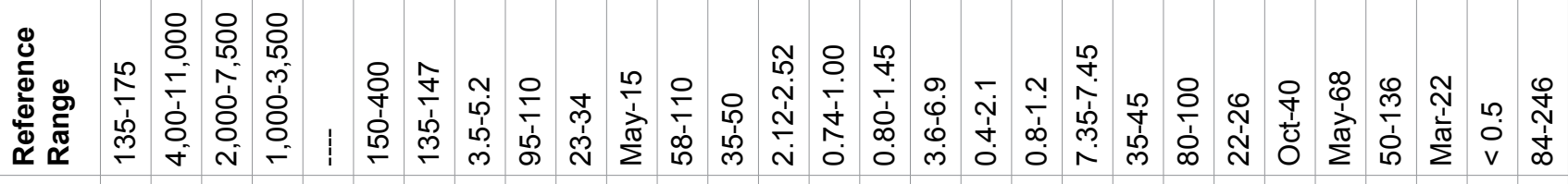

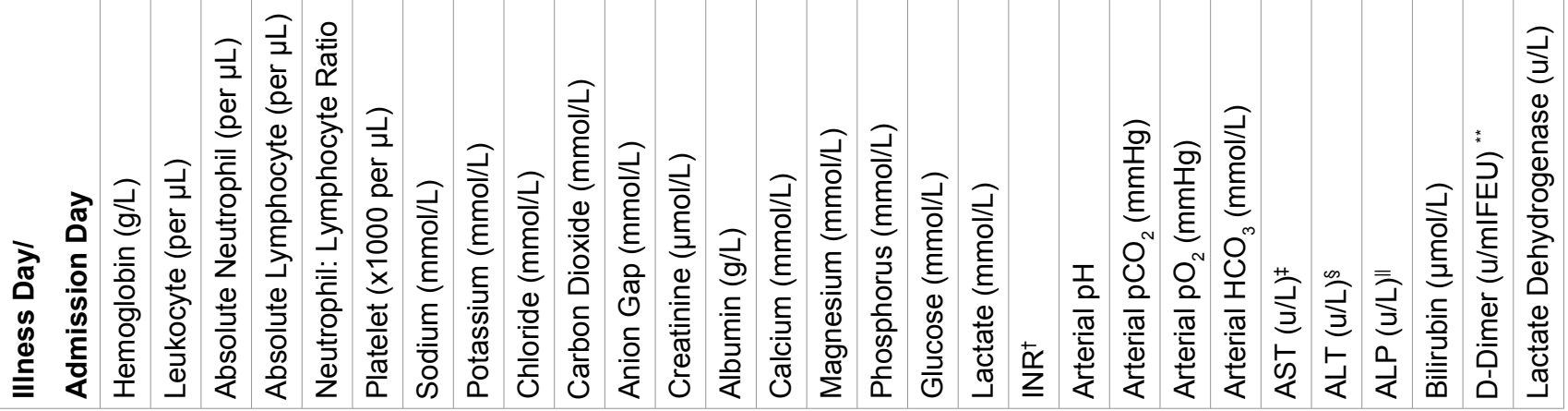


$\square$

$11 \frac{8}{2}$

11

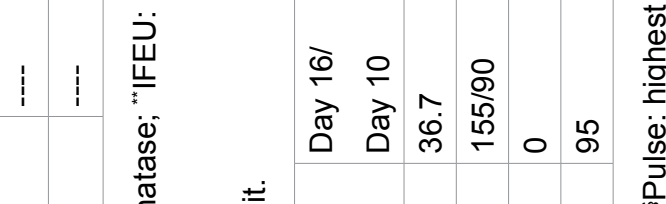

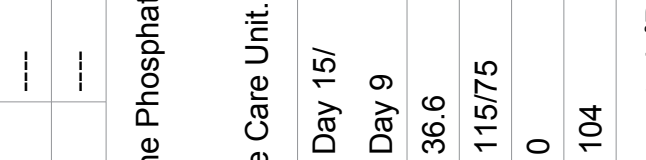

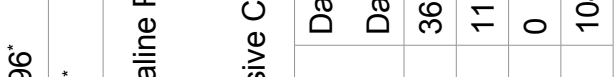

ঙู่

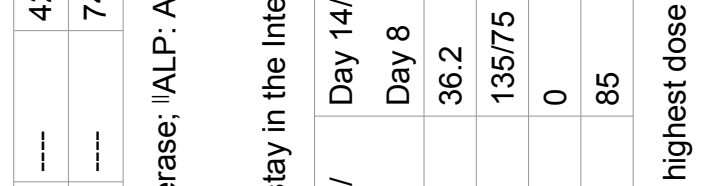

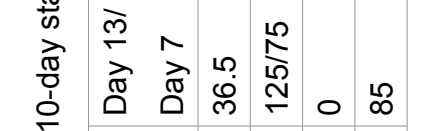

11 高

11 安

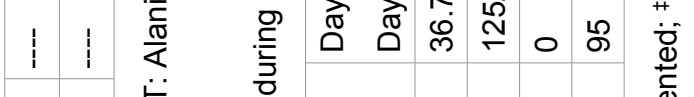

至

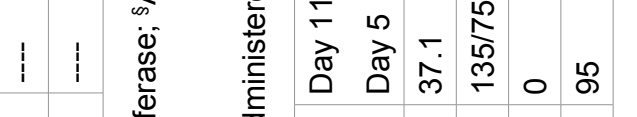

$\frac{\bar{\omega}}{\omega} \quad \frac{\varepsilon}{\sigma}$

1 |

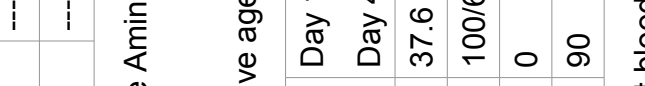

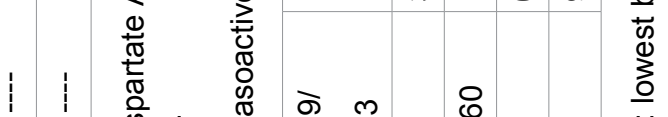

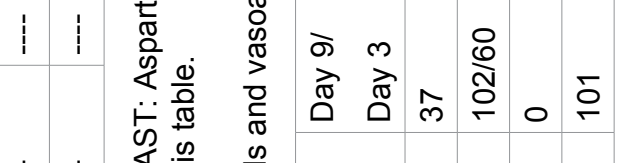

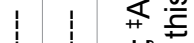

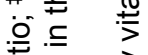

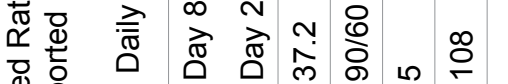

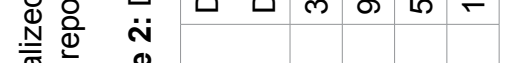

จ $\stackrel{\infty}{\infty}$

$\stackrel{\sim}{\mathrm{V}} \stackrel{\infty}{\sim}$

$\frac{2}{\pi} \frac{\pi}{\frac{\pi}{0}}$

읗

产范

כ)

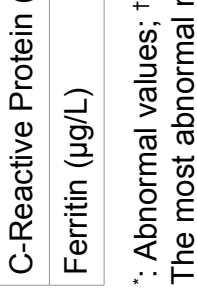

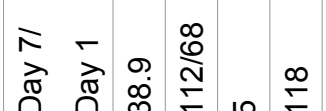

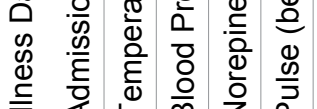

\section{䓂}

离

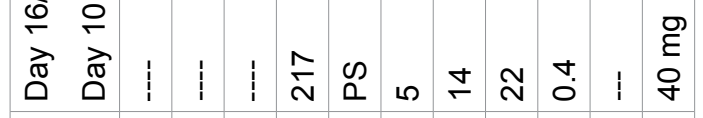

के

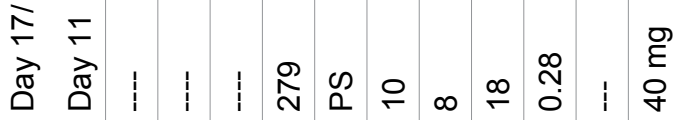

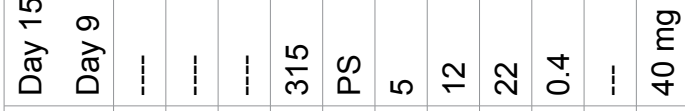

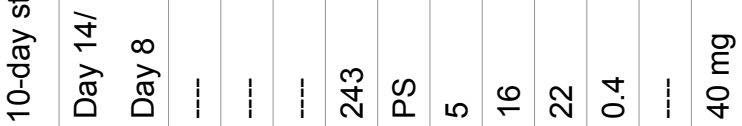

胥

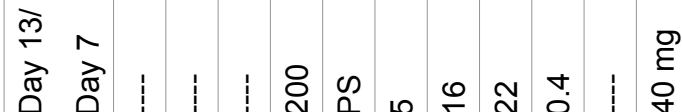
帛

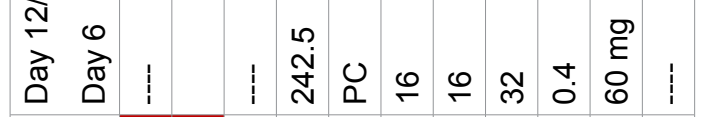

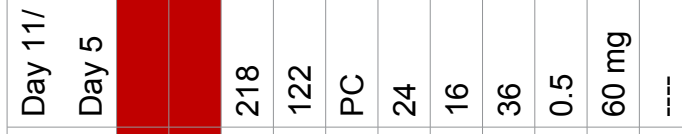

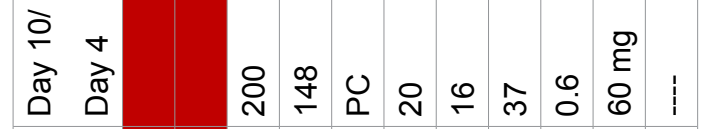

일

$\frac{\bar{m}}{\bar{m}}$

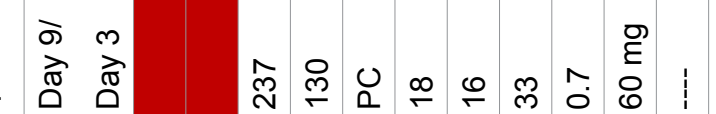

(s)

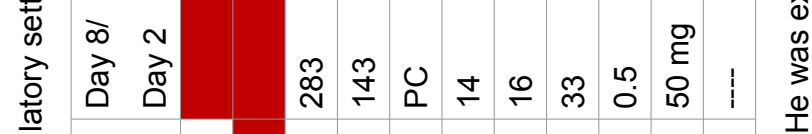

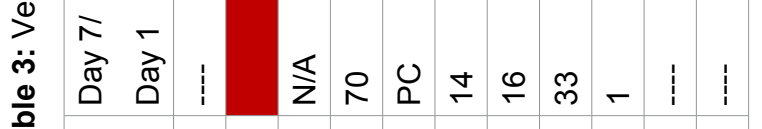

亮

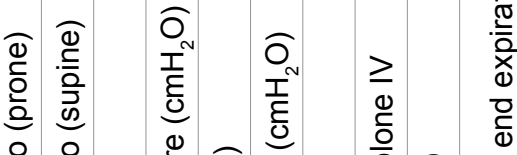

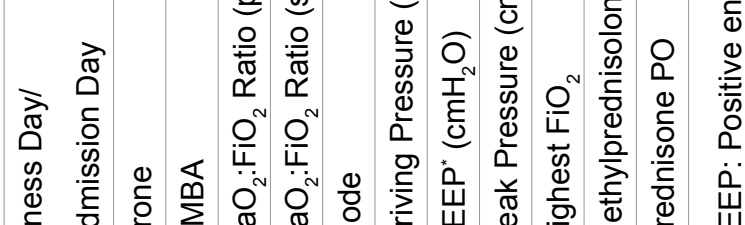




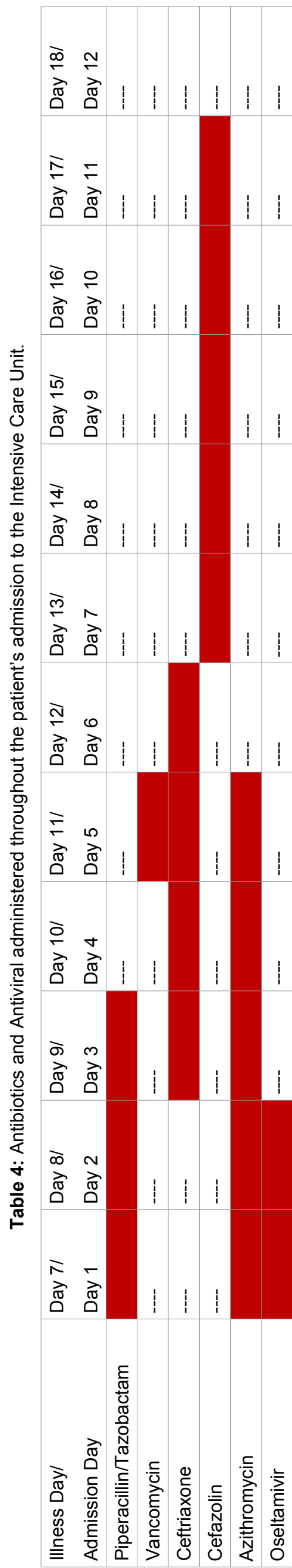

vere hypoxic respiratory failure. Early proning has been established in the ARDS literature to decrease mortality most prominently from the PROSEVA trial, a landmark multi-center randomized control trial [8]. The results have been reinforced by subsequent meta-analysis [9] for use in moderate to severe ARDS. Approximately 5\% of patients with COVID-19 infection progress to critical illness with respiratory failure [3] with imaging showing progression from diffuse bilateral ground glass opacities to consolidation and mixed pattern [10]. This pattern is consistent with ARDS and suggests that same physiological mechanism for improving oxygenation applies with proning. Complications of proning are notably that of endotracheal tube (ETT) obstruction [9]. In our practice, difficulty included the lack of familiarity with a proning protocol and the number of personnel required. Due to the risk of accidental extubation or disconnection of the ventilator circuit [11], our institution has regarded this procedure as an aerosol generating medical procedure. This raises a practical implications with increased amounts of personal protective equipment (PPE) required, during a time of PPE shortage [12]. There can be a significant delay in therapy if ETT obstruction occurs due to time required for PPE donning. Currently the SSC guidelines suggest the use of proning for 12-16 hours for moderate to severe ARDS in COVID-19 patients [4].

Our patient received a course of systemic corticosteroid for severe ARDS. There is currently conflicting evidence on use of systemic corticosteroids in ventilated patients with COVID-19, and ARDS. Early evidence from China from retrospective analysis suggests decreased risk of death, and improved oxygenation with corticosteroids $[13,14]$. Previous literature on SARS-CoV 2003 and MERS-CoV showed increased and prolonged viral load with the use of corticosteroids $[15,16]$. Corticosteroid usage in severe influenza was also associated with increased mortality and infections [17]. Overall, the current SSC recommendation is against the use of routine steroids in COVID-19. However, in the setting of ARDS, they recommended the use of corticosteroids [4].

Various clinicians and researchers have noted that SARS-CoV-2 binds to target cells through Angiotensin Converting Enzyme 2 (ACE2) and that the expression of ACE2 is increased in patients with diabetes, and those treated with ACE inhibitors and ARBs [18]. Theoretically, treatment with these agents may increase the risk of developing severe COVID-19 [18]. However, other studies have also illustrated that higher levels of ACE2 are protective against ARDS, and ARBs upregulate the activity of ACE2 [19]. Therefore, despite theoretical concerns, the Canadian Cardiovascular Society support the continuation of RAAS (renin-angiotensin-aldosterone system) inhibitors in otherwise stable patients with COVID-19 [20].

A point of interest is that our patient's neutrophil:lymphocyte ratio was elevated on admission to 
hospital and remained elevated. One study has used a neutrophil: lymphocyte ratio of $>3.13$ as a risk factor for severe ARDS [21]. This warrants further investigation to predict which patients with COVID-19 may go on to respiratory failure and need closer monitoring.

Currently, there are no effective antiviral or immunomodulatory therapies for the treatment of COVID-19 infection of any severity, including ARDS. The largest randomized trial to date using lopinavir/ritonavir did not find any difference between the two arms. However, the timing of administration of the study drug was thought to be too late to alter disease outcomes [22]. Another clinical trial made headlines regarding the use of combined azithromycin and hydroxychloroquine. However, the limitations of this trial include non-randomization, non-blinding and a large proportion of patients lost to follow-up [23]. There are several trials being launched in Canada, the largest of these being the "Multi-center, Adaptive, Randomized, Open-label, Controlled Clinical Trial of the Safety and Efficacy of Investigational Therapeutics for the Treatment of COVID-19 in Hospitalized Patients (CATCO)", which will use lopinavir/ ritonavir vs. hydroxychloroquine vs. remdesivir vs. optimized supportive care. Another large randomized trial, "Anti-Coronavirus Therapies to prevent progression of COVID-19 (ACT)" will use a combination of chloroquine and azithromycin. Both trials will include critically ill patients with ARDS.

There is still only early studies and indirect evidence to guide therapy on critically ill patients with COVID-19. This case outlines our early experience at a community hospital using currently available guidelines, specifically in the management of severe ARDS secondary to COVID-19. Ongoing studies are needed such as ACT and CATCO to expedite research on effective treatment.

\section{Ethics Approval and Consent to Participate}

Informed consent was obtained from the patient and the Hamilton Integrated Research Ethics Board (REB) has waived the requirement of REB approval.

\section{Consent for Publication}

We obtained verbal consent by discussing the consent in person with the patient in the presence of a bedside registered nurse as a witness. The patient was too weak to actually sign the consent. Due to the pandemic and restricted visiting policy, we were not able to obtain a written consent from his substitute decision maker (SDH) either. However, we have obtained telephone consent from his SDM as well. A copy of the consent form obtained verbally is available for review by the Editor of this journal.

\section{Availability of Data and Materials}

Not applicable.

\section{Competing Interests}

None to declare.

\section{Funding}

None.

\section{Authors' Contributions}

JT: Patient care, data collection, drafted the case, coordinated the case study with other authors, finalized the manuscript, acted as the corresponding author.

HC: Drafted background, literature review, reviewed manuscript, collected data.

GF: Drafted background, literature review, reviewed manuscript.

\section{DF: Daily data collection, reviewed manuscript.}

MK: Literature review, formatted tables and figures, reviewed manuscript.

All authors read and approved the final manuscript.

\section{Acknowledgement}

I would like to thank all the frontline healthcare providers who contributed to the care of this patient.

\section{References}

1. Lu R, Zhao X, Li J, Niu P, Yang B, et al. (2020) Genomic characterisation and epidemiology of 2019 novel coronavirus: implications for virus origins and receptor binding. Lancet 395: 565-574.

2. Coronavirus COVID-19 Global Cases by the Center for Systems Science and Engineering (CSSE). Johns Hopkins University (JHU).

3. Wu Z, McGoogan JM (2020) Characteristics of and important lessons from the Coronavirus disease 2019 (COVID-19) outbreak in China: Summary of a report of 72314 cases from the Chinese centre for disease control and prevention. JAMA 323.

4. Alhazzani W, Møller MH, Arabi YM, Loeb M, Gong MN, et al. (2020) Surviving Sepsis Campaign: Guidelines on the management of critically ill adults with Coronavirus Disease 2019 (COVID-19). Intensive Care Med.

5. Walkey AJ, Goligher EC, Sorbo LD, Hodgson CL, Adhikari NKJ, et al. (2017) Low tidal volume versus non-volume-limited strategies for patients with acute respiratory distress syndrome. A systematic review and meta-analysis. Ann Am Thorac Soc14: S271-S279.

6. Yasuda H, Nishimura T, Kamo T, Sanui M, Nango E, et al. (2017) Optimal plateau pressure for patients with acute respiratory distress syndrome: A protocol for a systematic review and meta-analysis with meta-regression. Bmj Open 7: e015091.

7. Slutsky AS, Ranieri VM (2013) Ventilator-Induced Lung Injury. New Engl J Medicine 369: 2126-2136.

8. Guérin C, Reignier J, Richard J-C, Beuret P, Gacouin A, et al. (2013) Prone positioning in severe acute respiratory distress syndrome. New Engl J Medicine 368: 2159-2168.

9. Bloomfield R, Noble DW, Sudlow A (2015) Prone position for acute respiratory failure in adults. Cochrane Db Syst Rev 11.

10. Shi H, Han X, Jiang N, Cao Y, Alwalid O, et al. (2020) Ra- 
diological findings from 81 patients with COVID-19 pneumonia in Wuhan, China: A descriptive study. Lancet Infect Dis 20: $425-434$.

11. Chatte G, Sab JM, Dubois JM, Sirodot M, Gaussorgues P, et al. (1997) Prone position in mechanically ventilated patients with severe acute respiratory failure. Am J Resp Crit Care 155: 473-478.

12. (2020) OMA Urges Governments To Distribute Personal Protective Equipment And Critical Care Supplies Now.

13. Wu C, Chen X, Cai Y, Xia J, Zhou X, et al. (2020) Risk factors associated with acute respiratory distress syndrome and death in patients with Coronavirus Disease 2019 Pneumonia in Wuhan, China. JAMA Intern Med 180.

14. Wang Y, Jiang W, He Q, Wang C, Wang B, et al. (2020) Early, low-dose and short-term application of corticosteroid treatment in patients with severe COVID-19 pneumonia: single-center experience from Wuhan, China. Medrxiv 20032342.

15. Lee N, Chan KCA, Hui DS, Ng EKO, Wu A, et al. (2004) Effects of early corticosteroid treatment on plasma SARS-associated Coronavirus RNA concentrations in adult patients. J Clin Virol 31: 304-309.

16. Arabi YM, Mandourah Y, Al-Hameed F, Sindi AA, Almekhlafi GA, et al. (2018) Corticosteroid therapy for critically ill patients with Middle East Respiratory Syndrome. Am J Resp Crit Care 197: 757-767.
17. Lansbury LE, Rodrigo C, Leonardi-Bee J, Nguyen-VanTam J, Lim WS (2020) Corticosteroids as adjunctive therapy in the treatment of Influenza: An Updated Cochrane Systematic Review and Meta-analysis. Crit Care Med 48.

18. Fang L, Karakiulakis G, Roth M (2020) Are patients with hypertension and diabetes mellitus at increased risk for COVID-19 infection? Lancet Respir Medicine 8.

19. Fedson DS, Opal SM, Rordam OM (2020) Hiding in plain sight: An approach to treating patients with Severe COVID-19 Infection. Mbio 11: e00398-e00420.

20. Krahn A, Bewick D, Chow C-M, Clarke B, Cowan S, et al. (2020) Guidance from the CCS COVID-19 Rapid Response Team.

21. Liu J, Liu Y, Xiang P, Pu L, Xiong H, et al. (2020) Neutrophil-to-Lymphocyte ratio predicts severe illness patients with 2019 Novel Coronavirus in the Early Stage. Medrxiv 20021584

22. Cao B, Wang Y, Wen D, Liu W, Wang J, et al. (2020) A trial of Lopinavir-Ritonavir in adults hospitalized with severe Covid-19. New Engl J Med.

23. Gautret $P$, Lagier J-C, Parola P, Hoang VT, Meddeb L, et al. (2020) Hydroxychloroquine and azithromycin as a treatment of COVID-19: Results of an open-label non-randomized clinical trial. Int J Antimicrob Ag 105949. 